


\title{
Finding Oxygen Reservoir by Using Extremely Small Test Cell Structure for Resistive Random Access
}

Received: 26 June 2015

Accepted: 17 November 2015

Published: 22 December 2015

\section{Memory with Replaceable Bottom Electrode}

\author{
Kentaro Kinoshita ${ }^{1,2,3}$, Sang-Gyu Koh ${ }^{1}$, Takumi Moriyama ${ }^{1,2}$ \& Satoru Kishida ${ }^{1,2,3}$
}

Although the presence of an oxygen reservoir (OR) is assumed in many models that explain resistive switching of resistive random access memory (ReRAM) with electrode/metal oxide (MO)/electrode structures, the location of $O R$ is not clear. We have previously reported a method, which involved the use of an AFM cantilever, for preparing an extremely small ReRAM cell that has a removable bottom electrode (BE). In this study, we used this cell structure to specify the location of OR. Because an anode is often assumed to work as OR, we investigated the effect of changing anodes without changing the MO layer and the cathode on the occurrence of reset. It was found that the reset occurred independently of the catalytic ability and Gibbs free energy $(\Delta G)$ of the anode. Our proposed structure enabled to determine that the reset was caused by repairing oxygen vacancies of which a filament consists due to the migration of oxygen ions from the surrounding area when high $\Delta G$ anode metal is used, whereas by oxidizing the anode due to the migration of oxygen ions from the MO layer when low $\Delta G$ anode metal is used, suggesting the location of $O R$ depends on $\Delta G$ of the anode.

Models in which resistive switching of an electrode/metal oxide (MO)/electrode structure is caused by the thermally activated migration of oxygen ions are being widely accepted. In these models, a conductive filament (CF) that is formed in the MO layer consists of oxygen vacancies, and resistive switching is caused by the generation and repair of oxygen vacancies $\left(\mathrm{V}_{\mathrm{O}}{ }^{\prime}\right)^{1-9}$. This means that resistive switching is caused by exchanging oxygen ions between the (CF) and the oxygen reservoir (OR). However, information about the OR is still incomplete. We do not even know the location of the OR; for example, is it located in the electrode or in the MO layer? The location of the OR should be elucidated to design the cell structure, including electrodes and MO materials for optimizing memory performance. Because many papers claim that an anode works as an $\mathrm{OR}^{10-13}$, it is advantageous for identifying the location of an OR if the anode can be replaced without changing the MO layer and the cathode.

Recently, we reported a method, which involved the use of an AFM cantilever, for preparing an extremely small ReRAM cell that has a removable bottom electrode $(\mathrm{BE})^{14}$. First, a MO layer is deposited on the surface of a Pt-coated cantilever that works as a top electrode (TE). Next, by contacting a BE with the cantilever, a tiny ReRAM cell structure is formed in the contact area. This method enables anode replacement at any time by simply moving the cantilever from one BE to another BE.

In this study, the influence of changing the anode material without changing the MO layer and the cathode of the cathode/ $\mathrm{MO} /$ anode structure on the presence and absence of a reset was investigated by utilizing our proposed cell structure. As a result, the MO layer is suggested to be the OR when a metal with a high Gibbs energy of formation reaction is used as the anode, whereas to be the anode itself when a metal with a low Gibbs energy is used as the anode.
${ }^{1}$ Department of Information and Electronics, Graduate School of Engineering, Tottori University, 4-101 KoyamaMinami, Tottori 680-8552, Japan. ${ }^{2}$ Tottori Integrated Frontier Research Center, 4-101 Koyama-Minami, Tottori 6808552, Japan. ${ }^{3}$ Tottori Univ. Electronic Display Research Center, 4-101 Koyama-Minami, Tottori 680-8552, Japan. Correspondence and requests for materials should be addressed to K.K. (email: kinoshita@ele.tottori-u.ac.jp) 
(a)



(b)

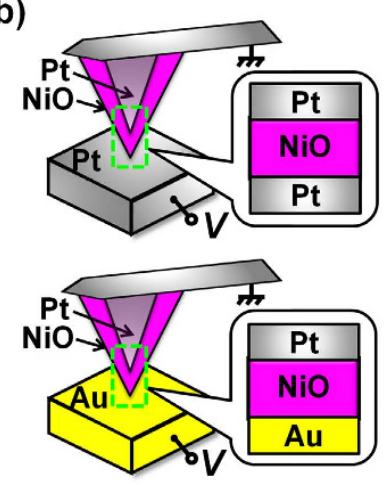

(c)

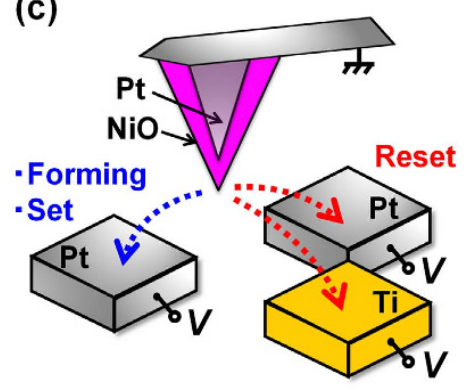

Figure 1. (a) A Pt-, Au-, Ni-, and TiN-BE, which were used only for reset, and another common Pt-BE, which was used only for forming and set, were formed on the same substrate. (b) Schematics explaining that the ReRAM cell is formed at the contact area between the cantilever and the BE. (c) A Ti- and Pt-BE, which were used only for reset, and another common Pt-BE, which was used only for forming and set, were formed on the same substrate.

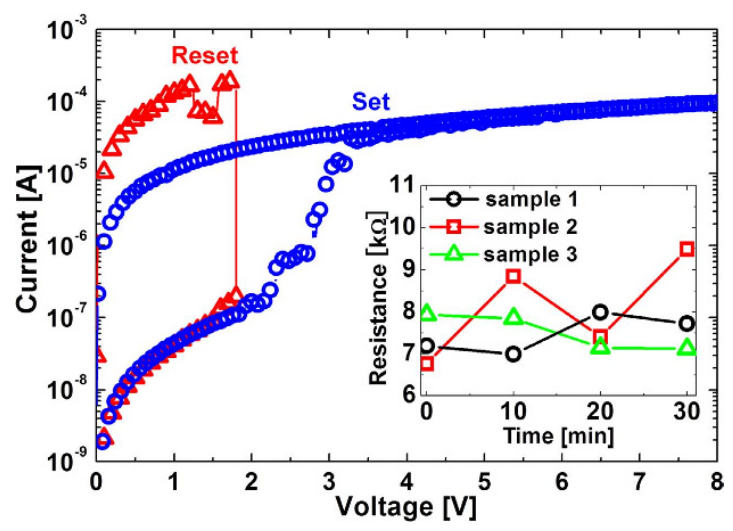

Figure 2. I- $V$ characteristics of a Pt-TE/NiO/Pt-BE structure that was measured by voltage sweep. Time dependences of resistances in the low resistance state for three different samples are shown in the inset.

\section{Results and Discussion}

Cantilevers on which a $\mathrm{Pt} / \mathrm{NiO}$ and an $\mathrm{Au} / \mathrm{NiO}$ structure were formed were prepared and will be described respectively as a Pt cantilever and an Au cantilever hereafter. On the other hand, Pt-, Au-, Ni-, and TiN-BE(R)s with a thickness of $100 \mathrm{~nm}$ were prepared on the same $\mathrm{SiO}_{2} / \mathrm{Si}$ substrate, as shown in Fig. 1(a). A Pt-TE/NiO/X-BE $(\mathrm{X}=\mathrm{Pt}$, $\mathrm{Au}, \mathrm{Ni}$, or TiN) structure was formed by contacting the $\mathrm{X}-\mathrm{BE}$ with the cantilever and the area of the structure was estimated to be less than $10 \mathrm{~nm}$ in diameter ${ }^{14}$. For example, by contacting the Pt-BE with the Pt cantilever, a $\mathrm{Pt}-\mathrm{TE} / \mathrm{NiO} / \mathrm{Pt}-\mathrm{BE}$ structure is formed in the contact area, as shown in the upper schematic in Fig. 1(b). On the other hand, by contacting the $\mathrm{Au}-\mathrm{BE}$ with the $\mathrm{Pt}$ cantilever, a Pt-TE/NiO/Au-BE structure is formed in the contact area, as shown in the lower schematic in Fig. 1(b). These four BEs were used for reset, and will be denoted hereafter as $\mathrm{X}-\mathrm{BE}(\mathrm{R})(\mathrm{X}=\mathrm{Pt}, \mathrm{Au}, \mathrm{Ni}$, or TiN). On the other hand, another common Pt-BE was also prepared on the same substrate for forming and set, and will be denoted hereafter as Pt-BE(FS). In addition, another substrate on which a Ti-BE for reset (Ti-BE(R)), Pt-BE(R), and Pt-BE(FS) was formed was also prepared, as shown in Fig. 1(c).

Figure 2 shows the current-voltage $(I-V)$ characteristics of a Pt-TE/NiO/Pt-BE structure that was measured by sweeping the bias voltage. Unipolar switching was confirmed, where set and reset occurred on Pt-BE(FS) and $\mathrm{Pt}-\mathrm{BE}(\mathrm{R})$, respectively. We investigated the dependence of $\mathrm{BE}$ materials on the occurrence of reset by applying pulse voltages to reduce the damage due to excess Joule heating. In addition, time dependences of resistances in the low resistance state for three different samples are shown in the inset of Fig. 2. Circles, squares, and triangles show time dependences of resistances for three different samples which were prepared under the same conditions. Although the low resistance state is retained at least more than $30 \mathrm{~min}$, the resistance randomly changes with the drift of cantilever, suggesting that the measurement conditions continuously change in a strict sense. Therefore, we avoid discussing the electrode material dependence of the resistance and the switching voltage, and restrict ourselves to discussion about the occurrence of resistive switching. The results of the investigation are presented later in the paper.

First, the cantilever was brought into contact with the Pt-BE(FS) in the atmosphere. After forming on the Pt-BE(FS), the cantilever was brought into contact with the Pt-BE(R), and the occurrence of the first reset was confirmed. Next, the chamber was evacuated to a pressure of $10^{-4} \mathrm{~Pa}$, and all the BEs were annealed for $10 \mathrm{~min}$ at 
(a)

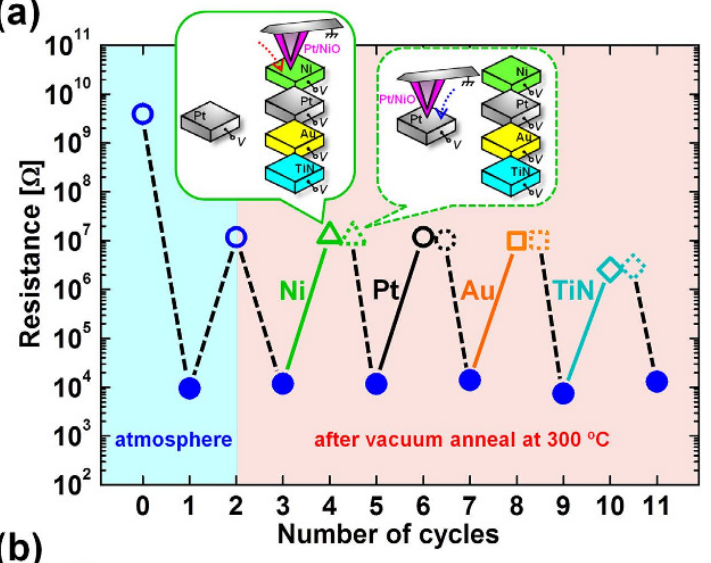

(b)



Figure 3. (a) Sequential resistive switching using the Pt cantilever: forming on $\mathrm{Pt}-\mathrm{BE}(\mathrm{FS}) \Rightarrow$ reset on $\mathrm{Pt}-\mathrm{BE}(\mathrm{R}) \Rightarrow$ vacuum anneal for $10 \mathrm{~min}$ at $300^{\circ} \mathrm{C} \Rightarrow$ set on $\mathrm{Pt}-\mathrm{BE}(\mathrm{FS}) \Rightarrow$ reset on $\mathrm{Ni}-\mathrm{BE}(\mathrm{R}) \Rightarrow$ set on Pt$\mathrm{BE}(\mathrm{FS}) \Rightarrow$ reset on $\mathrm{Pt}-\mathrm{BE}(\mathrm{R}) \Rightarrow$ set on $\mathrm{Pt}-\mathrm{BE}(\mathrm{FS}) \Rightarrow$ reset on $\mathrm{Au}-\mathrm{BE}(\mathrm{R}) \Rightarrow$ set on $\mathrm{Pt}-\mathrm{BE}(\mathrm{FS}) \Rightarrow$ reset on TiN $-B E(R)$, where all the measurements after the vacuum annealing were performed at RT without breaking the vacuum. Resistance values after set and reset are shown respectively by filled and open symbols. After the occurrence of reset on each $\mathrm{BE}(\mathrm{R})(\mathrm{Ni}$ : open triangle, Pt: open circle, Au: open square, TiN: open diamond), resistance value was read out again on the Pt-BE(FS), and the readout value are shown by dottedline symbols. (b) Sequential resistive switching using the Au cantilever: forming on $\mathrm{Pt}-\mathrm{BE}(\mathrm{FS}) \Rightarrow$ reset on $\mathrm{Pt}-\mathrm{BE}(\mathrm{R}) \Rightarrow$ vacuum anneal for $10 \mathrm{~min}$ at $300^{\circ} \mathrm{C} \Rightarrow$ set on $\mathrm{Pt}-\mathrm{BE}(\mathrm{FS}) \Rightarrow$ reset on $\mathrm{Au}-\mathrm{BE}(\mathrm{R}) \Rightarrow$ set on $\mathrm{Pt}$ $\mathrm{BE}(\mathrm{FS}) \Rightarrow$ reset on $\mathrm{TiN}-\mathrm{BE}(\mathrm{R}) \Rightarrow$ set on $\mathrm{Pt}-\mathrm{BE}(\mathrm{FS}) \Rightarrow$ reset on $\mathrm{Pt}-\mathrm{BE}(\mathrm{R}) \Rightarrow$ set on $\mathrm{Pt}-\mathrm{BE}(\mathrm{FS}) \Rightarrow$ reset on $\mathrm{Ni}$ $-B E(R)$, where all the measurements after the vacuum annealing were performed at RT without breaking the vacuum. Resistance values after set and reset are shown respectively by filled and open symbols. After the occurrence of reset on each $\mathrm{BE}(\mathrm{R})$ (Au: open square, TiN: open diamond, Pt: open circle, Ni: open triangle), resistance value was read out again on the Pt-BE(FS), and the readout value are shown by dotted-line symbols.

$300^{\circ} \mathrm{C}$ in order to desorb water from the surface of the BEs to avoid field oxidation or reduction of the NiO layer and the BEs. After cooling the BEs to room temperature (RT), the cantilever was brought into contact with the $\mathrm{Pt}-\mathrm{BE}(\mathrm{FS})$ again, and the occurrence of the first set was confirmed. Resistances before forming, after forming, after the first reset, and after the first set are denoted by the left four circles in Fig. 3(a), where resistance values read out after set (forming) and reset are shown respectively by filled and open symbols. Next, the cantilever was brought into contact with $\mathrm{Ni}-\mathrm{BE}(\mathrm{R})$, and the occurrence of reset was confirmed. Next, the cantilever was brought into contact with Pt-BE(FS), and the occurrence of set was confirmed. In the same way, reset was attempted sequentially on each BE in the order of Ni-, Pt-, Au-, and TiN-BE(R) using the same cantilever at RT without breaking the vacuum. All the set processes were performed on the Pt-BE(FS) as described earlier. Reset was confirmed on all the BEs and, therefore, high resistance values were read out after the occurrence of reset as shown respectively by the open triangle, circle, square, and diamond in Fig. 3(a). Average pulse heights at which reset occurred were 1.43, $1.62,1.48$, and $1.13 \mathrm{~V}$ for Ni-, Pt-, Au-, and TiN-BE(R), respectively. After the occurrence of reset on each BE(R), the resistance value was read out again on the Pt-BE(FS), and the readout values are shown by the dotted-line symbols in Fig. 3(a). The high resistance values observed on the $\mathrm{BE}(\mathrm{R})$ s were retained after moving the cantilever to $\mathrm{Pt}-\mathrm{BE}(\mathrm{FS})$.

The same measurement was performed by using a Au-cantilever instead of using a Pt-cantilever. The result is shown in Fig. 3(b). In this case, we also observed the occurrence of reset on all of the $\mathrm{BE}(\mathrm{R})$ s and the high resistance values observed on the $\mathrm{BE}(\mathrm{R}) \mathrm{s}$ were retained after moving the cantilever to Pt-BE(FS) as well as the case using 


\begin{tabular}{|c|c|c|c|}
\hline & Reset? & $\begin{array}{c}\text { Catalytic } \\
\text { ability }\end{array}$ & $\begin{array}{l}\text { Gibbs energy } \\
: \Delta \mathrm{G}[\mathrm{kJ} / \mathrm{mol}]\end{array}$ \\
\hline $\mathrm{Pt}\left(\mathrm{PtO}_{2}\right)$ & \multirow{4}{*}{ Yes (filament) } & Yes & 166.9 \\
\hline $\mathrm{Au}\left(\mathrm{Au}_{2} \mathrm{O}_{3}\right)$ & & No & 77.9 \\
\hline $\mathrm{Ni}(\mathrm{NiO})$ & & Yes & -211.5 \\
\hline $\mathrm{Ti}(\mathrm{TiN})$ & & No & -309.2 \\
\hline $\mathrm{Ti}\left(\mathrm{TiO}_{2}\right)$ & Yes (anode) & No & -883.3 \\
\hline
\end{tabular}

Table 1. The presence of catalytic ability and Gibbs free energy of formation reaction $(\Delta G)$ at room temperature of the bottom electrodes (BEs) used in this study, where $\Delta \mathrm{G}$ is defined as the difference between the Gibbs free energy of metal oxide or metal nitride indicated in the parenthesis and the Gibbs free energy of pure metal ( $\mathrm{Pt}, \mathrm{Au}, \mathrm{Ni}$, and $\mathrm{Ti})$.

the Pt-cantilever. Average pulse heights at which reset occurred were 1.15, 1.70, 1.20, and 1.33 V for Ni-, Pt-, Au-, and $\mathrm{TiN}-\mathrm{BE}(\mathrm{R})$, respectively.

We discuss these results in terms of catalysis and Gibbs free energy of formation reaction $(\Delta G)$ of the BEs, where $\Delta \mathrm{G}$ is defined as the difference between the Gibbs free energy of $\mathrm{MO}\left(\mathrm{PtO}_{2}, \mathrm{Au}_{2} \mathrm{O}_{3}, \mathrm{NiO}\right.$, and $\left.\mathrm{TiO}_{2}\right)$ or metal nitride $(\mathrm{TiN})$ and the Gibbs energy of pure metal (Pt, $\mathrm{Au}, \mathrm{Ni}$, and $\mathrm{Ti}$ ). The presence of catalytic ability (in terms of the dissociation of oxygen) and $\Delta \mathrm{G}$ at RT of the BEs are summarized in Table $1^{15}$. In addition to the presence of water on the BEs as described earlier, we have to consider some other absorbing species depending on BE materials. We focus on oxygen and the hydroxyl radical $(\mathrm{OH})$ as possible absorbing species that enhance reset switching, because reset is generally thought to be caused by repairing a part of the $\mathrm{V}_{\mathrm{O}}$ 's that a CF comprises. Oxygen is known to be dissociatively-adsorbed on the surface of $\mathrm{Pt}$, and the desorption temperatures of molecular and atomic oxygen from the $\mathrm{Pt}(111)$ surface are reported respectively to be 150 and $750 \mathrm{~K}^{16}$. On the other hand, $\mathrm{OH}$ is reported to be formed following the reaction formula ${ }^{17,18}$ :

$$
\mathrm{H}_{2} \mathrm{O}(\mathrm{a})+\mathrm{O}(\mathrm{a}) \rightarrow 2 \mathrm{OH}(\mathrm{a})
$$

where $\mathrm{H}_{2} \mathrm{O}(\mathrm{a}), \mathrm{O}(\mathrm{a})$, and $\mathrm{OH}(\mathrm{a})$ mean $\mathrm{H}_{2} \mathrm{O}, \mathrm{O}$, and $\mathrm{OH}$ absorbed on the surface of Pt. However, the desorption temperature of $\mathrm{H}_{2} \mathrm{O}$ is reported to be approximately $210 \mathrm{~K}$, and thus no $\mathrm{OH}$ is present on the surface of Pt-BE after vacuum annealing for $10 \mathrm{~min}$ at $300^{\circ} \mathrm{C}(=573 \mathrm{~K})^{17}$. Therefore, we have to consider the presence of atomic oxygen among oxidizing species. In addition, no catalytic effect on oxygen has been reported on the surfaces of $\mathrm{Au}$ and TiN. Therefore, our result suggests that reset occurs independently of the presence or absence of catalytic ability of anodes, suggesting that the formation of atomic oxygen with the support of the catalysis of Pt-BE is not crucial. It is also noted that the result shown in Fig. 3(b) suggests that catalytic ability of cathodes is not necessary for the occurrence of reset.

On the other hand, significant results were observed when a Ti-BE(R) was used. We performed the same experiment by using a substrate on which a Ti-BE(R) was formed with Pt-BE(FS) and Pt-BE(R), which is shown in Fig. 1(c). The $\Delta \mathrm{G}$ of Ti is shown in Table 1. After confirming the occurrence of forming and the first reset on the Pt-BEs in the atmosphere as shown respectively by the first filled circle and second open circle, both the Ptand Ti-BEs were annealed in vacuum for $10 \mathrm{~min}$ at $300^{\circ} \mathrm{C}$ and cooled to RT. The occurrence of the first set was observed as shown by filled circle in Fig. 4 . Next, the cantilever was moved to the Ti-BE, and reset was attempted. The occurrence of reset was confirmed on the Ti-BE(R) at an average pulse height of $0.80 \mathrm{~V}$ and, therefore, the readout value of the resistance was high enough to be recognized as a high resistance state (HRS) as shown by the open triangle in Fig. 4. However, this high resistance value was not maintained when the cantilever was moved to the Pt-BE(FS) without applying a voltage. The readout value of the resistance on the Pt-BE(FS) was low enough to be judged as a low resistance state (LRS), as shown by the dotted-line triangle in Fig. 4.

This series of results suggest that when an anode with a high $\Delta \mathrm{G}$ is used, reset occurs owing to the migration of oxygen ions inside the $\mathrm{NiO}$ layer, whereas when an anode with a low $\Delta \mathrm{G}$ is used, reset occurs owing to oxidation of the anode. Therefore, the NiO layer itself works as an OR for anodes with a high $\Delta \mathrm{G}$, whereas the anode itself works as an OR for anodes with a low $\Delta \mathrm{G}$. It is widely accepted that CFs consist of $V_{O}$ 's, and reset is caused by $\mathrm{V}_{\mathrm{O}}$ migration due to the $\mathrm{V}_{\mathrm{O}}$ concentration gradient. On the basis of this reset mechanism, set could occur owing to the electric field drift of $V_{O}$ 's when an anode with a low $\Delta \mathrm{G}$ is used. In this case, a reset and set are caused by the exchanging of $\mathrm{V}_{\mathrm{O}}$ 's between the $\mathrm{CF}$ and the anode in a direction perpendicular to the electrode interface ${ }^{12}$. However, the anode with a $\Delta \mathrm{G}$ that is much lower than the $\Delta \mathrm{G}$ of Ni in anode/ $\mathrm{NiO} /$ cathode structures deprives oxygen from the $\mathrm{NiO}$ layer. As a result, highly insulating oxide is formed at the surface of the anode and prevents the occurrence of resistive switching ${ }^{19}$. On the other hand, because the OR was suggested to be an $\mathrm{NiO}$ layer itself when an anode with a high $\Delta \mathrm{G}$ is used as an anode, set and reset are to be repeated by exchanging $V_{O}$ 's between a $\mathrm{CF}$ and an $\mathrm{NiO}$ layer excluding the CF. Therefore, a driving force that is different from an electric field drift and that works in a direction parallel to the electrode interface is required to cause set switching when an anode with a high $\Delta \mathrm{G}$ is used. One of the candidates for this driving force is the Soret force, which works in the direction of the temperature gradient ${ }^{20-24}$. In this case, resistive switching takes place simply by the modification of the $V_{O}$ distribution inside the $\mathrm{NiO}$ layer and, therefore, good switching endurance is ensured. 


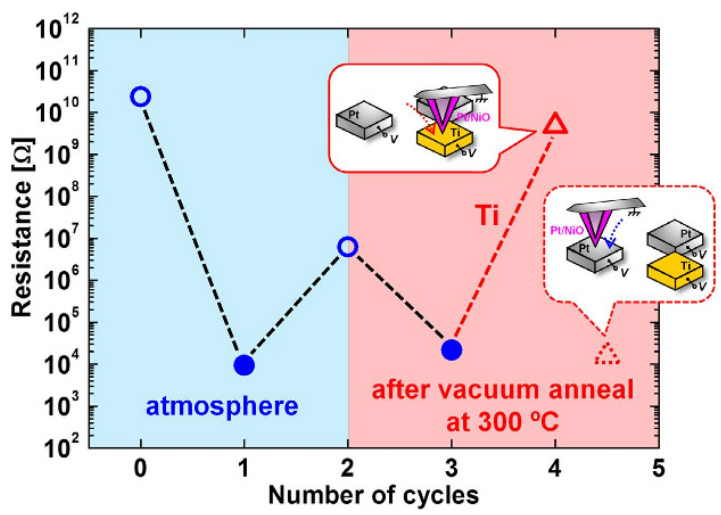

Figure 4. Sequential resistive switching: forming on $\mathrm{Pt}-\mathrm{BE}(\mathrm{FS}) \Rightarrow$ reset on $\mathrm{Pt}-\mathrm{BE}(\mathrm{R}) \Rightarrow$ vacuum anneal for 10 min at $300^{\circ} \mathrm{C} \Rightarrow$ set on $\mathrm{Pt}-\mathrm{BE}(\mathrm{FS}) \Rightarrow$ reset on $\mathrm{Ti}-\mathrm{BE}(\mathrm{R}) \Rightarrow$ set on $\mathrm{Pt}-\mathrm{BE}(\mathrm{FS})$, where all the measurements after the vacuum annealing were performed at $R T$ without breaking the vacuum. Resistance values after set and reset are shown respectively by filled and open symbols. After the occurrence of reset on the Ti-BE(R) (open triangle), resistance value was read out again on the Pt-BE(FS), and the readout value is shown by dottedline triangle.

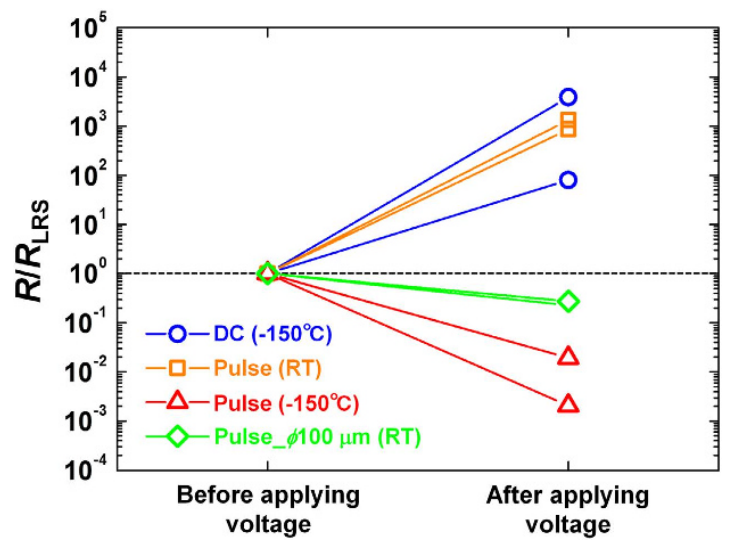

Figure 5. Resistance before and after the attempt to reset $\mathrm{Pt}$ cantilever/Pt-BE(R) structures by ramping voltage from 0 up to $3 \mathrm{~V}$ with sweep rate of $\sim 5 \mathrm{~V} / \mathrm{s}$ at $-150^{\circ} \mathrm{C}$ (circles) and by applying voltage pulse with rise time of $2 \mu \mathrm{s}$, which corresponds to sweep rate of $\sim 10^{6} \mathrm{~V} / \mathrm{s}$, at room temperature (squares) and $-150^{\circ} \mathrm{C}$ (triangles). Diamonds show resistance before and after the attempt to reset normal $\mathrm{Pt} / \mathrm{NiO} / \mathrm{Pt}$ stack structures with the area of $100 \mu \mathrm{m}$ in diameter by applying a voltage pulse with the rise time of $2 \mu \mathrm{s}$ at room temperature.

Figure 5 shows resistance before and after the attempt to reset $\mathrm{Pt}$ cantilever/Pt-BE(R) structures by ramping the voltage up from 0 to $3 \mathrm{~V}$ with a sweep rate of $\sim 5 \mathrm{~V} / \mathrm{s}$ and by applying a voltage pulse with a rise time of $2 \mu \mathrm{s}$, which corresponds to a sweep rate of $\sim 10^{6} \mathrm{~V} / \mathrm{s}$. The occurrence of reset was confirmed both after applying the sweep voltage at $-150^{\circ} \mathrm{C}$ (circle in Fig. 5) and after applying the voltage pulse at RT (square in Fig. 5) as well as after applying the sweep voltage at RT as shown in Fig. 2. On the other hand, reset did not occur after applying the voltage pulse at $-150^{\circ} \mathrm{C}$ (triangles in Fig. 5). The resistance became rather low compared with the resistance before applying the voltage pulse. This result can be explained by assuming that the driving force causing set switching is the Soret force. The fluxes of oxygen vacancies for Fick diffusion $J_{\text {Fick }}$ and for Sorret diffusion $J_{\text {Soret }}$ can be expressed as

$$
\left\{\begin{array}{l}
J_{\text {Fick }} \approx-D_{\mathrm{V}} \frac{\mathrm{d} n_{V}}{\mathrm{~d} x}, \\
J_{\text {Soret }} \approx-D_{\mathrm{V}} S_{\mathrm{V}} n_{\mathrm{V}} \frac{d T}{d x},
\end{array}\right.
$$

where $D_{\mathrm{V}}, n_{\mathrm{V}}$, and $S_{\mathrm{V}}$ are the diffusion constant, the density, and the Soret coefficient of the vacancies as a function of the radial coordinate, $x$, respectively, if a cylindrical cell structure is assumed. Here, the Soret coefficient is defined as the ratio of the thermal diffusion constant and the normal diffusion constant ${ }^{25}$ and must be negative for $\mathrm{V}_{\mathrm{O}} \mathrm{s}^{24}$. Assuming that the $\mathrm{CF}$ consists of $\mathrm{V}_{\mathrm{O}}{ }^{\prime 26,27}$, they diffuse from the $\mathrm{CF}$ to the outside of the CF by Fick diffusion because $\mathrm{V}_{\mathrm{O}}$ 's diffuse from high- to low- $\mathrm{V}_{\mathrm{O}}$ concentration areas according to Equation 2, generating $J_{\text {Fick }}$ and causing reset switching. On the other hand, $\mathrm{V}_{\mathrm{O}}$ 's diffuse toward a current path that is a heating center owing 




Figure 6. Simulated temperature distribution in $\mathrm{Pt} / \mathrm{NiO} / \mathrm{Pt}$ stack structures with (a) large (10 $\mu \mathrm{m}$ in diameter) and (b) small (100 $\mathrm{nm}$ in diameter) area, respectively, at $1.01 \mathrm{~V}$ during application of a voltage pulse with the rise time of $2 \mu \mathrm{s}$ and the pulse height of $1.20 \mathrm{~V}$. A filament consisting of $\mathrm{V}_{\mathrm{O}}$ 's with the radius of $10 \mathrm{~nm}$ is located at the center before the voltage application in both large and small structures. $n_{\mathrm{V}}$ distribution in (c) large and (d) small structures for the temperature distributions (a) and (b), respectively.

to Joule heating from the surrounding area by Soret diffusion because $\mathrm{V}_{\mathrm{O}}$ 's diffuse from low- to high-temperature areas according to Equation 3, generating $J_{\text {Soret }}$ and causing set switching. So, $J_{\text {Fick }}$ and $J_{\text {Soret }}$ are always competing during resistive switching. When the voltage sweep rate is small enough to maintain thermal equilibrium continuously, $J_{\text {Soret }}$ is small because the temperature gradient, $\mathrm{d} T / \mathrm{dx}$, in Equation 3 is small. However, when the voltage sweep rate becomes large so that thermal equilibrium cannot be maintained, the larger the voltage sweep rate becomes, the larger $\mathrm{d} T / \mathrm{d} x$ becomes. $J_{\text {Soret }}$, therefore, becomes dominant for larger voltage sweep rates. In addition, because $S_{\mathrm{Vo}}$ is expressed as $-U /\left(k_{\mathrm{B}} T^{2}\right)$, the ratio of $J_{\text {Fick }}$ to $J_{\text {Soret }}$ is proportional to $T^{2}$, i.e., $\left|J_{\text {Fick }} / J_{\text {Soret }}\right| \propto T^{2}$, where $U$ is the energy barrier between the potential wells for $\mathrm{V}_{\mathrm{O}}$ diffusion, and $k_{\mathrm{B}}$ is the Boltzmann constant ${ }^{24}$. This means that $J_{\text {Soret }}$ becomes dominant at low temperatures and hinders reset switching. Therefore, it is expected that it will be difficult for reset to occur when a voltage pulse with a short rise time is applied at a low temperature, which is consistent with the result shown in Fig. 5.

On the other hand, we also measured resistance before and after attempt to reset normal $\mathrm{Pt} / \mathrm{NiO} / \mathrm{Pt}$ stack structures with the area of $100 \mu \mathrm{m}$ in diameter by applying a voltage pulse with the rise time of $2 \mu \mathrm{s}$ and the pulse height of $1.2 \mathrm{~V}$ at RT (diamonds in Fig. 5). In this case, reset did not occur and the resistance rather decreased in contrast to the case using Pt cantilever/Pt-BE(R) structures at RT. Figure 6(a,b) show simulated temperature distribution in $\mathrm{Pt} / \mathrm{NiO} / \mathrm{Pt}$ stack structures with large $(10 \mu \mathrm{m}$ in diameter $)$ and small $(100 \mathrm{~nm}$ in diameter) area, respectively, at $1.01 \mathrm{~V}$ during application of a voltage pulse with the rise time of $2 \mu \mathrm{s}$ and the pulse height of $1.20 \mathrm{~V}$ (Fig. S2). A CF consisting of $\mathrm{V}_{\mathrm{O}}$ 's with the radius of $10 \mathrm{~nm}$ is located at the center before the voltage application (for details, see supplemental information). Temperature gradient near the CF is steep in the large structure, whereas relatively flat in the small structure due to the accumulation of Joule heat. As a result, in the large structure, oxygen vacancies diffuse toward the center of the CF by Soret diffusion as shown in Fig. 6(c), causing set switching. On the other hand, in the small structure, oxygen vacancies diffuse outward from the CF by Fick diffusion, and oxygen vacancy concentration is flattened as shown in Fig. 6(d), causing reset switching. Therefore, the simulation result is consistent with the experimentally obtained data above, supporting that Soret diffusion is the driving force of set switching.

In conclusion, the present work provided a picture of the occurrence of resistive switching: the location of the OR differs depending on the $\Delta \mathrm{G}$ of the anode material, and resistive switching occurs by the exchange of $\mathrm{V}_{\mathrm{O}}$ 's between a CF and the OR. An OR is a NiO layer excluding the $\mathrm{CF}$ for high $\Delta \mathrm{G}$ values, whereas it is an anode for low $\Delta \mathrm{G}$ values. This clearly suggests that the location of the OR depends on the relative magnitude of the Gibbs energies of the anode material and the metal $\mathrm{M}$ of an $\mathrm{MO}$ layer. The presence of a driving force in a direction parallel to the electrode interface is required to cause set switching when an anode with a high $\Delta \mathrm{G}$ is used. Elucidating the driving force is crucial for a deeper understanding of the resistive switching mechanism.

\section{Methods}

Sample preparation. A Pt or Au film with a thickness of $20 \mathrm{~nm}$ was deposited on an AFM cantilever with a tip radius of $50 \mathrm{~nm}$ (Hitachi High-Technologies, SI-DF3-R(100)) as the TE, followed by the 
deposition of an $\mathrm{NiO}$ film with a thickness of $15 \mathrm{~nm}$ as a memory layer at RT by using the DC reactive magnetron sputtering method in a mixture of $\mathrm{Ar}+\mathrm{O}_{2}$ gases. During the $\mathrm{NiO}$ deposition, the pressure of the mixture gas was maintained at $0.50 \mathrm{~Pa}\left(\mathrm{Ar}: \mathrm{O}_{2}=0.42: 0.08 \mathrm{~Pa}\right)$. The cantilever on which a $\mathrm{Pt} / \mathrm{NiO}$ or an $\mathrm{Au} / \mathrm{NiO}$ structure was formed was described respectively as a Pt cantilever or an Au cantilever in this paper. We also prepared normal $\mathrm{Pt}(100 \mathrm{~nm}) / \mathrm{NiO}(60 \mathrm{~nm}) / \mathrm{Pt}(100 \mathrm{~nm})$ stack structures. $100 \mathrm{~nm}$ thick Pt-TEs with the area of $100 \mu \mathrm{m}$ in diameter were deposited by DC sputtering using a shadow mask, after NiO deposition by DC reactive sputtering method at $380^{\circ} \mathrm{C}$ in the mixed gas of $\mathrm{Ar}$ and $\mathrm{O}_{2}$ gasses $\left(\mathrm{Ar}: \mathrm{O}_{2}=0.45 \mathrm{~Pa}: 0.05 \mathrm{~Pa}\right)$.

Pt-BE(FS) and Pt-, Au-, Ni-, and TiN-BE(R)s with a thickness of $100 \mathrm{~nm}$ were prepared on the same $\mathrm{SiO}_{2}(100 \mathrm{~nm}) / \mathrm{Si}(650 \mu \mathrm{m})$ substrate by a sputtering method (Fig. 1(a)). In addition, Pt-BE(FS) and Ti- and Pt-BE(R) $s$ with a thickness of $100 \mathrm{~nm}$ were prepared on the another same $\mathrm{SiO}_{2}(100 \mathrm{~nm}) / \mathrm{Si}(650 \mu \mathrm{m})$ substrate by a sputtering method (Fig. 1(c)). This substrate was annealed under a reductive atmosphere of $\mathrm{H}_{2}$ and Ar mixture gas (3.0\% of $\mathrm{H}_{2}$ ) for $5 \mathrm{~min}$ at $300^{\circ} \mathrm{C}$ for the reduction of the native oxide film that was formed on the surface of Ti-BE(R).

Electric characteristics measurements. A bias voltage was applied between the cantilever and the BE by using a pulse generator (Agilent 81110A) or a source measure unit (Keythley 236) in the contact mode of the AFM. A reset was attempted by applying pulse voltages with the duration of $100 \mu \mathrm{s}$. The pulse height was increased from $0.8 \mathrm{~V}$ in steps of $0.1 \mathrm{~V}$ until a voltage was reached at which the occurrence of reset was confirmed. On the other hand, forming and set were performed by sweeping the voltage to $10 \mathrm{~V}$ and to $8 \mathrm{~V}$, respectively. By sequentially contacting each $\mathrm{BE}$ with the same cantilever, the effect of replacing anodes on the resistive switching property was investigated. The bias voltage was applied to the BEs, whereas a cantilever that works as a TE was grounded. The bias voltage was always positive in this study: we attempted only unipolar switching. The AFM system used in this study (Hitachi High-Technologies, E-sweep) was equipped with a vacuum chamber and a heater in the chamber so that the atmospheric pressure and temperature were controllable. Forming and the first reset were performed on the Pt-BE(FS) and the Pt-BE(R) in the atmosphere, respectively. After that, all switching was performed in vacuum $\left(\sim 10^{-4} \mathrm{~Pa}\right)$. A substrate temperature was controlled within the range $-150^{\circ} \mathrm{C}$ to $300^{\circ} \mathrm{C}$.

Simulation. Reset switching was attempted by simulating $\mathrm{V}_{\mathrm{O}}$ migration using commercial software (COMSOL Multiphysics). We assumed Soret and Fick diffusion, whereas electric field drift and Fick diffusion are generally adopted as driving forces of $\mathrm{V}_{\mathrm{O}}$ migration. For the details about simulation, see supplemental information.

\section{References}

1. Kinoshita, K. et al. Bias polarity dependent data retention of resistive random access memory consisting of binary transition metal oxide. Appl. Phys. Lett. 89, 103509 (2006).

2. Tsunoda, K. et al. Low Power and High Speed Switching of Ti-doped NiO ReRAM under the Unipolar Voltage Source of less than 3 V. Tech. Dig.-Int. Electron Devices Meet. 2007, 767-770 (2007).

3. Park, G.-S. et al. Observation of electric-field induced Ni filament channels in polycrystalline $\mathrm{NiO}_{\mathrm{x}}$ film. Appl. Phys. Lett. 91, 222103 (2007).

4. Kim, K.-M., Choi, B.-J. \& Hwang, C.-S. Localized switching mechanism in resistive switching of atomic-layer-deposited $\mathrm{TiO}_{2}$ thin films. Appl. Phys. Lett. 90, 242906 (2007).

5. Sawa, A. Resistive switching in transition metal oxides. Mater. Today 11, 28-36 (2008)

6. Kwon, D.-H. et al. Atomic structure of conducting nanofilaments in $\mathrm{TiO}_{2}$ resistive switching memory. Nat. Nanotech. 5, 148-153 (2010).

7. Ielmini, D., Nardi, F. \& Cagli, C. Universal Reset Characteristics of Unipolar and Bipolar Metal-Oxide RRAM. IEEE Trans. Electron Devices 58, 3246-3253 (2011).

8. Yoshida, C., Kinoshita, K., Yamasaki, T. \& Sugiyama, Y. Direct observation of oxygen movement during resistance switching in NiO/ Pt film. Appl. Phys. Lett. 93, 042106 (2008).

9. Ninomiya, T. et al. Conductive Filament Scaling of $\mathrm{TaO}_{\mathrm{x}}$ Bipolar ReRAM for Improving Data Retention Under Low Operation Current. IEEE Trans. Electron Devices 60, 1384-1389 (2013).

10. Lee, M.-J. et al. Electrical Manipulation of Nanofilaments in Transition-Metal Oxides for Resistance-Based Memory. Nano Lett. 9, 1476-1481 (2009).

11. Akinaga, H. \& Shima, H. Resistive Random Access Memory (ReRAM) Based on Metal Oxides. Proc. IEEE 98, 2273-2251 (2010).

12. Yu, S. \& Wong, H.-S. P. A Phenomenological Model for the Reset Mechanism of Metal Oxide RRAM. IEEE Electron Device Lett. 31, 1455-1457 (2010).

13. Goux, L. et al. Role of the anode material in the unipolar switching of TiN/NiO/Ni cells. J. Appl. Phys. 113, 054505 (2013).

14. Koh, S.-G., Kishida, S. \& Kinoshita, K. Extremely small test cell structure for resistive random access memory element with removable bottom electrode. Appl. Phys. Lett. 104, 083518 (2014).

15. Barin, I. Thermochemical Data of Pure Substances 98-1547 (VCH, 1989).

16. Gland, J.-L. Molecular and atomic adsorption of oxygen on the $\operatorname{Pt}(111)$ and $\mathrm{Pt}(\mathrm{S})-12(111) \times(111)$ surfaces. Surf. Sci. 93, 487-514 (1980).

17. Creighton, J.-R. \& White, J.-M. A static sims study of $\mathrm{H}_{2} \mathrm{O}$ adsorption and reaction on clean and oxygen-covered $\mathrm{Pt}(111)^{+}$. Chem. Phys. Lett. 92, 435-438 (1982).

18. Michaelides, A. \& Hu, P. Catalytic Water Formation on Platinum: A First-Principles Study. Am. Chem. Soc. 123, 4235-4242 (2001).

19. Lee, C.-B. et al. Effects of metal electrodes on the resistive memory switching property of NiO thin films. Appl. Phys. Lett. 93, 042115 (2008).

20. Goldhirsch, I. \& Ronis, D. Theory of thermophoresis. I. General considerations and mode-coupling analysis. Phys. Rev. A 27, 1616-1634 (1983).

21. Ewart, F. et al. Oxygen potential measurements in irradiated mixed oxide fuel. J. Nucl. Mater. 124, 44-55 (1984).

22. Janek, J. \& Timm, H. J. Thermal diffusion and Soret effect in (U,Me) $\mathrm{O}_{2+\delta}$ : the heat of transport of oxygen. Nucl. Mater. 255, 116-127 (1998).

23. Kempers, L.-J.-T.-M. A comprehensive thermodynamic theory of the Soret effect in a multicomponent gas, liquid, or solid. J. Chem. Phys. 115, 6330-6341 (2001).

24. Strukov, D.-B., Alibart, F. \& Williams, R.-S. Thermophoresis/diffusion as a plausible mechanism for unipolar resistive switching in metal-oxide-metal memristors. Appl. Phys. A 107, 509-518 (2012)

25. Platten, J. K. The Soret Effect: A Review of Recent Experimental Results. J. Appl. Mechanics 73, 5 (2006). 
26. Bersuker, G. et al. Metal oxide resistive memory switching mechanism based on conductive filament properties. J. Appl. Phys. 110, 124518 (2011).

27. Sarhan, A. et al. Oxygen vacancy effects on electronic structure of Pt/NiO/Pt capacitor-like system. Surf. Sci. 606, 239-246 (2012).

\section{Acknowledgements}

A part of this work was supported by "Nanotechnology Platform Project (Nanotechnology Open Facilities in Osaka University)" of Ministry of Education, Culture, Sports, Science and Technology, Japan [No.:S-14-OS-0017].

\section{Author Contributions}

K.K. and S.-G.K. conceived and conducted the experiments. K.K., S.-G.K., T.M. and S.K. analyzed the results. All authors reviewed the manuscript.

\section{Additional Information}

Supplementary information accompanies this paper at http://www.nature.com/srep

Competing financial interests: The authors declare no competing financial interests.

How to cite this article: Kinoshita, K. et al. Finding Oxygen Reservoir by Using Extremely Small Test Cell Structure for Resistive Random Access Memory with Replaceable Bottom Electrode. Sci. Rep. 5, 18442; doi: 10.1038/srep18442 (2015).

(c) (i) This work is licensed under a Creative Commons Attribution 4.0 International License. The images or other third party material in this article are included in the article's Creative Commons license, unless indicated otherwise in the credit line; if the material is not included under the Creative Commons license, users will need to obtain permission from the license holder to reproduce the material. To view a copy of this license, visit http://creativecommons.org/licenses/by/4.0/ 